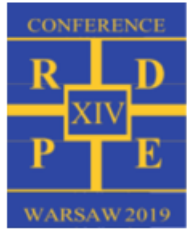

\title{
Experimental investigation of LWR passive safety systems performance at the INKA test facility
}

\author{
Rafat B ryk $^{1,2^{*}}$, Thomas $\mathrm{M} \mathrm{ull}^{2}$ and Holger Schmidt ${ }^{2}$ \\ ${ }^{1}$ Institute of Heat Engineering, Warsaw University of Technology, N ow owiejska 21/25, 00-665 W arsaw, Poland \\ 2 Framatome G mbH, Paul-Gossen-Straße 100, 91052 Erlangen, rafal.bryk@ framatome.com, Germany
}

\begin{abstract}
INKA is a test facility designed by Framatome and built in the technical center in Karlstein. The original objective for establishing this test rig was the investigation of the performance of the passive safety systems developed in a new Framatome Boiling Water Reactor (BWR) design - KERENA. INKA was constructed in the scale of 1:1 in heights while the total volume of the containment was replicated in 1:24. Since the geometries of particular safety systems are faithfully reflected, their actual performance in the original plant can be investigated at the full scale. Due to the unquestionable interest of the nuclear community in the inherent safety, not only new BWR and PWR designs are equipped with the passive systems, but also particular passive solutions are considered to be applied into the already existing Light Water Reactors (LWR). In this context and due to the fact that both, single component tests and integral tests can be conducted at INKA, the facility can be employed for a demonstration/qualification of a large range of passive safety systems foreseen for quite different types of LWRs. Hence, the goal of the EASY project was the experimental confirmation of the passive systems performance and the analysis of their interactions between each other in the integral tests. Besides, the overarching target of all tests performed at INKA is provision of data for codes validation. This paper presents major outcomes and conclusions drawn on the basis of EASY project results.
\end{abstract}

\section{Introduction}

Passive systems increase inherent safety of nuclear power plants by utilizing basic laws of physics such as gravity and natural circulation for removal of residual heat from the reactor pressure vessel (RPV) in case of an accident. Properly designed passive solutions enable bringing the plant to safe and stable condition without the need for active systems and corresponding engines, electric instrumentation and control equipment etc. This in turn decreases the total investment cost as well as lowers the maintenance effort. The concept of the passive safety eliminates also the human factor, which is considered to be the reason of the most relevant accidents in the nuclear industry.

Following the trend of the inherent safety increase and setting standards in this field at the same time, Framatome developed a new boiling water reactor (BWR) design - KERENA. KERENA is a medium size reactor equipped with both, passive and active systems. The development of the reactor was based on combining the state of the art knowledge from experiences collected during elaborating solutions for other products as well as on the implementation of new ideas [1].

In order to examine the performance of the passive systems of KERENA, a dedicated test facility was built at the Components Testing Department of Framatome in Karlstein, Germany. The Integral Test Facility Karlstein (INKA) represents the KERENA containment with a volume scaling of 1:24. Components heights and levels over the ground are kept in the full scale. The test rig setup allows to investigate both, each component individually and the whole system during integral tests. For the purpose of testing the integral system, INKA is equipped with several large vessels corresponding to the individual pools and vessels of KERENA. The RPV vessel has a storage capacity of $1 / 6$ of the KERENA RPV. In order to simulate the decay heat of the core, the vessel is fed with steam by the Benson boiler with maximum power output of $22 \mathrm{MW}$. The vessel is designed for operation pressure up to 160 bars. Compartments of the KERENA RPV are represented by separate vessels: flooding pool vessel, pressure suppression pool vessel and drywell vessel. An additional tank is used as a representation of the shielding/storage pool vessel [2]. Figure 1 illustrates replication of KERENA by the INKA test facility.

Since several passive systems are installed at the INKA facility and some solutions are utilized also in other light water reactors (LWR), the test facility itself can be perceived as a test rig for investigation of passive systems in general and not only as a one replicating a single reactor. This is particularly valid in the light of the possibility of investigation of individual components as well as interactions between them. Thus, for instance the passive pressure pulse transmitter (PPPT) which was analyzed at INKA is also considered as an actuator for the Safety Condenser (SACO) system for the pressurized water reactor (PWR) steam generators (SG). 


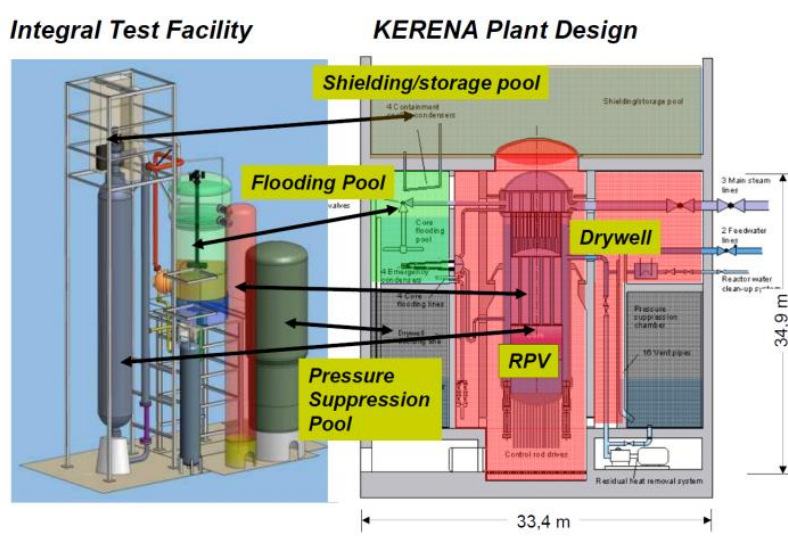

Fig. 1. KERENA replication by the INKA facility

Furthermore, besides the new BWR and PWR designs particular passive solutions are considered to be applied into already existing LWRs. In this context INKA can be employed for demonstration/qualification of a large range of passive safety systems foreseen for quite different types of LWRs. With this in mind, the Integral Experimental and Analytical Safety Cases for Design-Basis Accidents in Nuclear Power Plants Featuring Passive Safety Systems (EASY) project was initiated in M arch 2015. In the frames of the project four integral tests were conducted with the main objective of data base generation for behavior of and interaction between the different passive systems and components under realistic accident conditions. Besides, the overarching target of the project was provision of data for codes validation.

All tests performed in the frames of EASY project were conducted as integral tests, so not only the performance of individual components was investigated but also interactions between them and their influence on the entire system. In each test different accident scenario was simulated and the response of the facility was observed. The following events were covered by the tests campaign:

- Loss of Coolant Accident (LOCA) by a break of the $M$ ain Steam Line (MSL) with simulation of the decay heat.

- Simulation of Feedwater Line Break (FWLB) with taking into account the decay heat

- Simulation of RPV bottom leak with taking into account the decay heat

- Simulation of Station Blackout (SBO) with taking into account the decay heat

All the above tests showed a demonstrable reliability of the passive systems. They also provided a comprehensive data base for better understanding of the involved phenomena. The test data constitute a robust validation cases for thermal-hydraulic systems codes.

\section{Passive systems of KERENA and INKA}

KERENA reactor is equipped with several passive safety systems which are replicated in the full scale at the INKA facility. However, if the original plant comprises more than one set of the same devices, only one was built at the test facility. For example, although the
KERENA reactor is equipped with four Emergency Condensers (EC) and four Containment Cooling Condensers (CCC) only one EC and CCC were installed at the INKA. Figure 2 illustrates all passive systems of KERENA which were replicated at INKA.

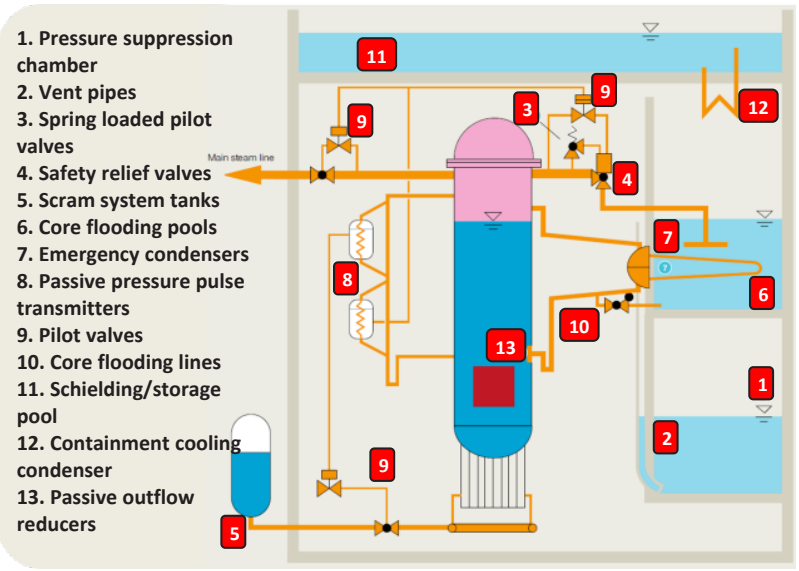

Fig. 2. Passive systems of the KERENA reactor

In case of an accident the reactor scram in the KERENA design is triggered by Passive Pressure Pulse Transmitters (PPPT). The PPPTs are small heat exchangers that operate according to the principle of communicating vessels (figure 3).

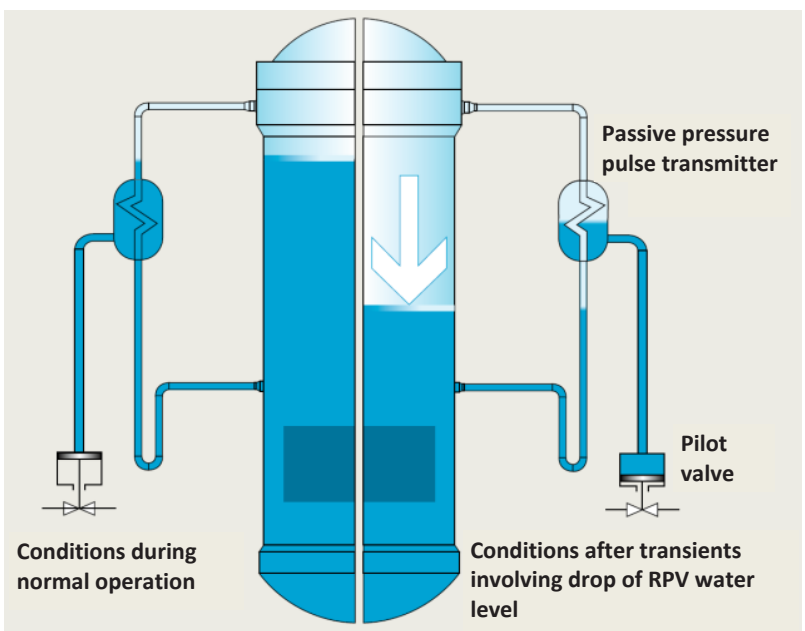

Fig. 3. PPPT operation

Upon a drop of the reactor water level, steam enters the top connective line and when it reaches the PPPT, pressure builds up on its secondary side due to the liquid expansion or evaporation caused by the temperature increase which in turn is a result of the heat transfer from the steam at the primary side. This pressure is then used to initiate safety-related switching operations without any need for electric power or manual or I\&C signals. PPPTs are installed in the KERENA reactor for safetyclassified initiation operations. Depending on the water level in the RPV, the PPPTs actuate different procedures (figure 2). Apart from reactor scram, they trigger automatic depressurization of the RPV by opening of the safety relief valves and they activate the isolation of the containment at the main steam lines. Their integration into the plant's systems engineering is (configuration: 
two 2-out-of-2) such that spurious actuation of a single PPPT does not lead to initiation of actions, but also such that loss of one PPPT cannot prevent initiation. PPPTs can be also applied to any kind of other systems triggered passively in other types of nuclear power plant. For instance PPPT is considered to serve as an actuator of the Safety Condenser (SACO) - passive system for heat removal from the PWR steam generators.

The Emergency Condenser (EC) system depressurizes and removes the decay heat from the RPV after the scram. The condenser is composed of a large number of slightly inclined tubes submerged in the core flooding pool and filled with cold water during normal operation of the plant (figure 4).

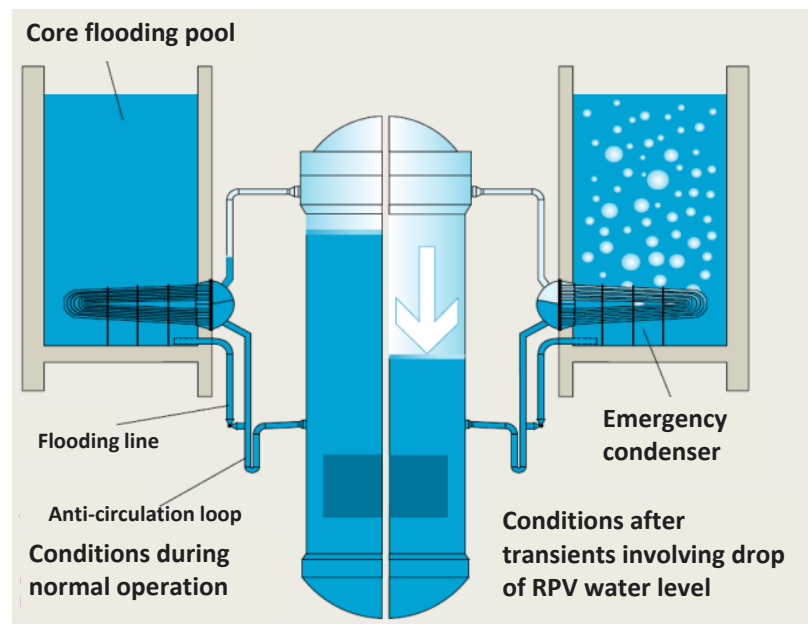

Fig. 4. EC operation [2]

Should the water level in the RPV decrease, the water drains from the tubes and the steam from the RPV enters the EC tubes. Due to the contact of external tube walls with the cold water in the core flooding pool, the steam at the primary side condenses leading to removal of substantial amount of heat. The heat transfer capacity of each EC is about $60 \mathrm{MW}$. Since the tubes of the EC are slightly inclined with respect to the horizontal orientation, the condensate flows back to the RPV due to gravity. The EC is connected with the RPV without any isolating elements and it is activated automatically without any need for electric power and according to the natural law of communicating vessels [2]. Therefore, since the EC system responses immediately to the liquid water drop in the RPV, the high pressure injection system is no longer necessary and it was eliminated from the KERENA design.

Due to its large capacity the Flooding Pool Vessel (FPV) acts not only as a sink for the EC but also for the Safety Relief Valve (SRV) system (figure 5).

The SRVs are of a diverse design: four of them operate according to the pressurization principle, four according to the pressure relief principle, thus excluding a loss of all safety relief valves due to a common mode failure.

The SRV system is located inside the containment and consists of the safety relief valves and relief lines with steam quenchers, which are installed in the core flooding pools. This system follows proven concepts used in the existing Framatome's BWR plants.

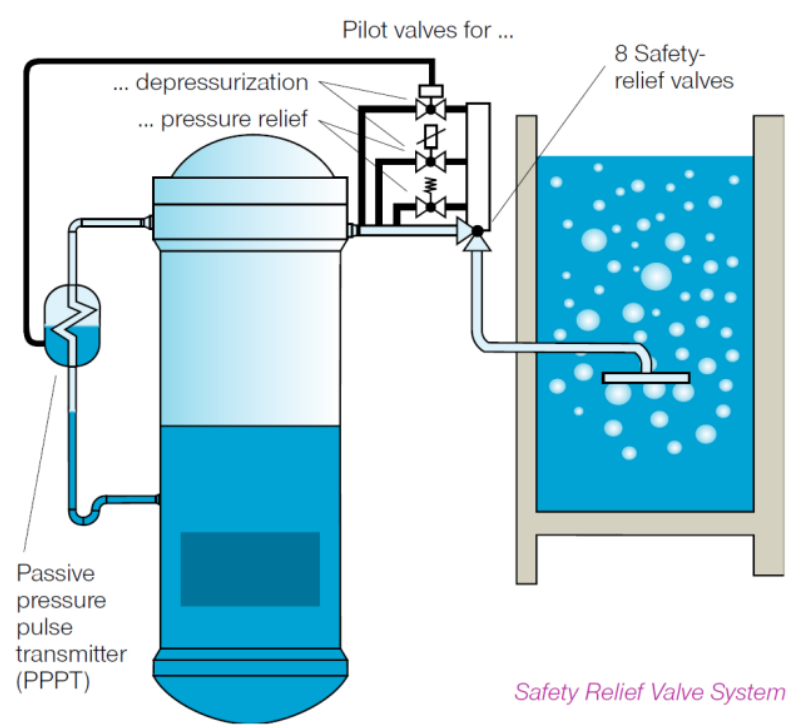

Fig. 5. Safety Relief $V$ alve operation

The FPV acts also as a water reservoir for passive core flooding. After the sufficient reduction of pressure in the RPV a check valve opens and it enables the water to flow gravitationally to the RPV (figure 6A). Despite the extremely low likelihood of a severe accident $(8.0 *$ $10^{-8}$ core damage frequency), the design of the KERENA reactor mitigates the consequences of such an event by employing the FPV also as a containment flooding pool. Therefore, even if it comes to the extremely improbable event of core meltdown, the core itself would remain in the RPV, which would be cooled-down from the external side (figure 6B).

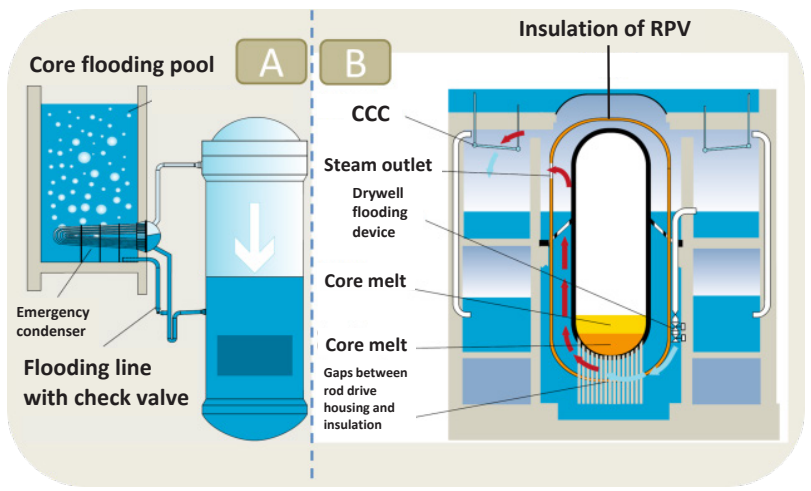

Fig. 6. RPV and containment flooding illustration

The Containment Cooling Condenser (CCC) is a system responsible for long-term (approximately 3 days) heat removal from the containment of the reactor. The whole CCC system consists of four heat exchangers hanged at the top of the containment vessel (Figure 2 and $6 \mathrm{~B})$. During normal operation of the reactor the condenser tubes are filled with cold water from the Shielding/Storage Pool Vessel (SSPV) and the CCC systems is not in operation. In case of a LOCA the steam could be released from the reactor loop increasing temperature and pressure inside the containment. Due to the temperature difference between the medium (steam- 
air mixture) in the containment and the water inside the $\mathrm{CCC}$ tubes, the heat transfer from the containment to the water in the tubes would be initiated (Figure 7).

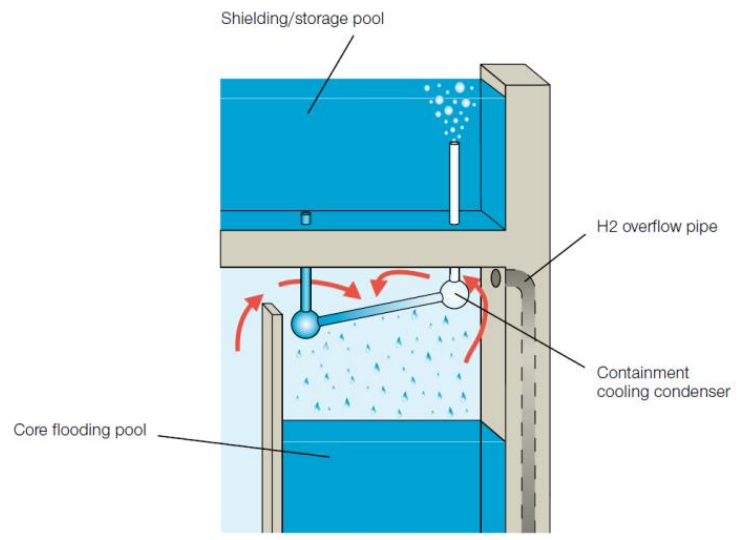

Fig. 7. CCC operation

Due to the density decrease resulting from the temperature increase in the CCC tubes the buoyancy force establishes natural circulation in the tubes. The fluid flow facilitates the heat removal to the long-term heat sink. At the primary side i.e. in the containment the steam released from the reactor loop condenses at the CCC tubes. Since condensation is a very efficient mechanism of heat transfer, substantial amount of heat can be removed from the containment. Furthermore, condensation at the $\mathrm{CCC}$ tubes supports the depressurization of the containment. Due to the placement of the CCCs at the top of the containment above the Flooding Pool Vessels, the condensate dribbles gravitationally from the CCC tubes to the FPV.

Depressurization and heat removal from the containment is also supported by the Pressure Suppression Pool (PSP). The PSP is a vessel filled with water up to a certain level and it is connected with the drywell with the vent pipe. Should the pressure in the containment increase, the water column in the vent pipes would be "pushed" downwards so that the steam released during a LOCA could enter the PSP and condense in the cold water in the pool. The PSP serves also as a water reservoir for the active residual heat removal system.

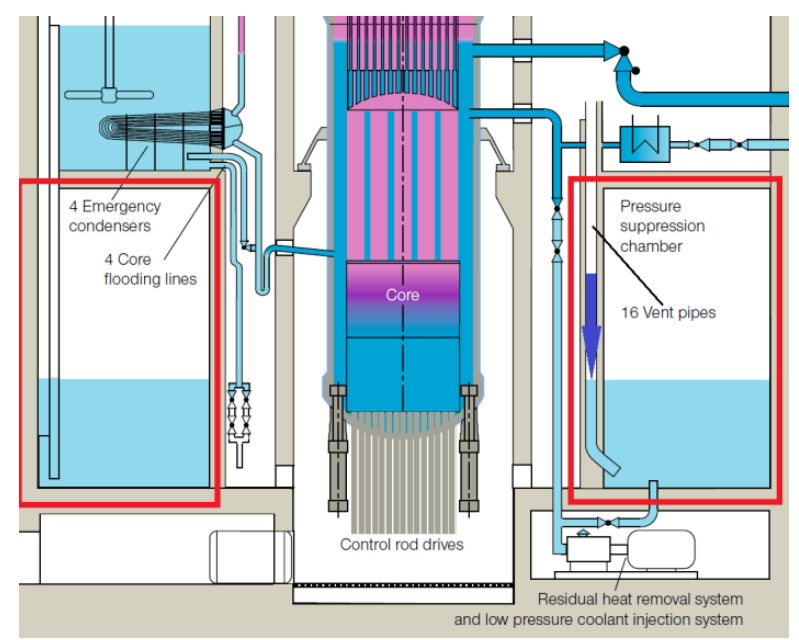

Fig. 8. PSPV operation

\section{INKA setup}

\subsection{Components arrangement}

The INKA test facility was designed to perform steadystate, full-scale tests of the passive systems (EC, CCC, passive core flooding, PPPT) and for simulating transient LOCA scenarios in a reduced scale $[3,4]$. Thus, the individual passive systems geometry at the INKA facility as well as the geodetical heights faithfully correspond to those at the KERENA reactor whereas the volumes of the containment are modeled to a scale of $1: 24$. The piping between the components was accurately designed for correct replication of those in the KERENA reactor. For a detailed determination of the heat transfer capacities as well as thermodynamic conditions in the individual components a dense network of instrumentation was provided.

The INKA facility is integrated into the large valve test facility Grossarmaturen-Prüfstand (GAP) that has been in operation in Karlstein for many years. The GAP test facility consists of a steam accumulator (height: 21.7 $\mathrm{m}$, volume: $125 \mathrm{~m}^{3}$ ) fed by a $22-\mathrm{MW}$ boiler. This accumulator supplies the steam needed for the experiments and replicates the RPV in the integral tests. An additional vessel with a water volume of about $50 \mathrm{~m}^{3}$ is placed inside the GAP support frame at the top to simulate the shielding/storage pool. This vessel supplies therefore water for the secondary side of the CCC.

The entire containment of the KERENA plant is simulated by three vessels. The Flooding Pool Vessel (FPV) represents the core flooding pools that contains the EC and the CCC. The Passive Core Flooding System connects the FPV with the return line of the EC. The PPPT is connected to the downcomer line. The Drywell Vessel (DWV) simulates the residual gas volume of the containment. The Pressure Suppression Pool (PSP) is simulated by a third vessel [3].

Figure 9 shows a simplified Piping and Instrumentation Diagram (P\&ID) of the INKA test facility with the containment. Two pipes connect the FPV with the DWV and represent the connections between the gas spaces of these compartments. The FPV is connected to the PSP vessel via the overflow pipe limiting the FPV water level. A second connection is the hydrogen overflow pipe used for pressure limitation during severe accident mitigation at KERENA. The DWV and the PSP vessel are connected via a full-scale vent pipe. Additionally, the function of the safety and relief valve is included in the design. The system is connected to the downcomer line and it goes into the FPV. For LOCA test scenarios, two different lines enter the Drywell Vessel, one for the simulation of breaks of a water line and the other for breaks of a steam line [3]. 


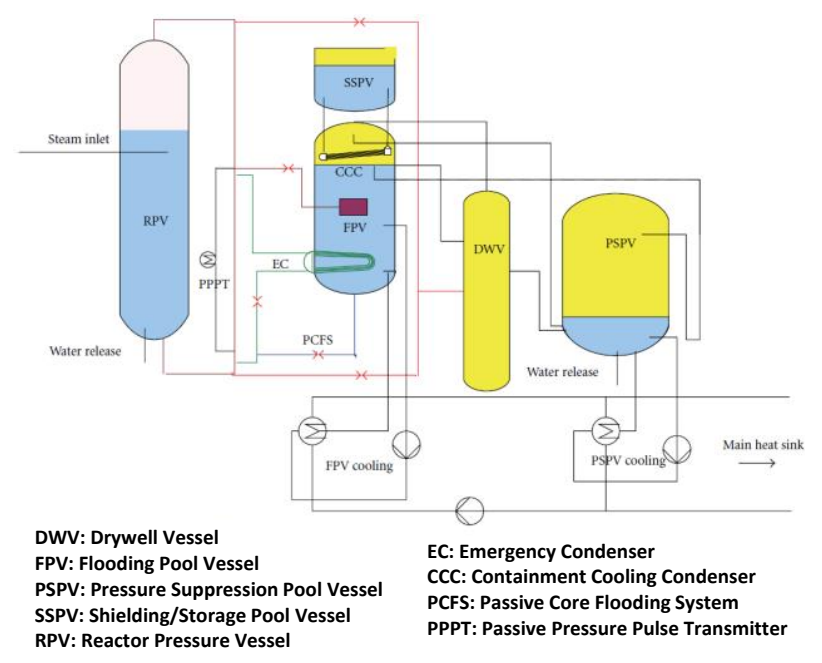

Fig. 9. Simplified P\&ID of the INK A facility [3]

\subsection{Instrumentation of the INKA facility}

The INKA facility is comprehensively instrumented with over 300 measurement sensors. Most of the instrumentation comprises widely used temperature, pressure, differential pressure and mass flow sensors. For two-phase flow measurement thermo-needle probes and gamma densitometer are installed at the INKA facility. The gas mixture in the vessels is measured via a probe sampling system analyzed by a mass spectrometer.

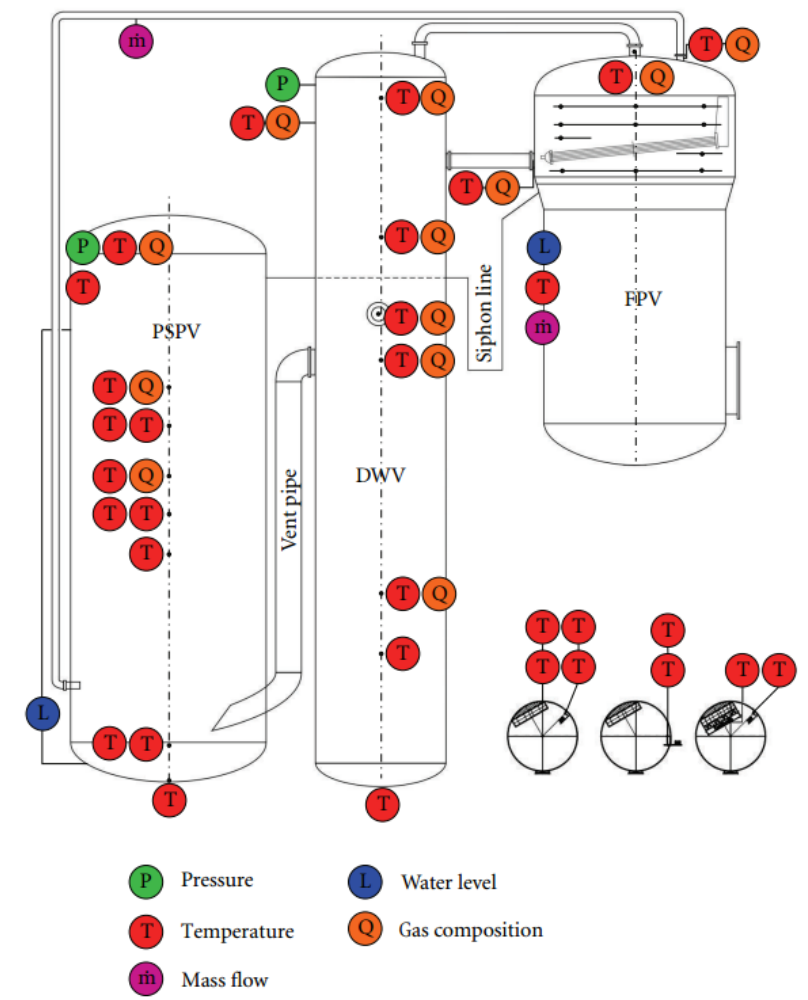

Fig. 10. INK A vessels instrumentation [3]

An overview of the INKA vessels (PSP, DWV and FPV) instrumentation is illustrated in the figure 10. For the obtainment of the complete information about the conditions inside the PSP, the vessel is instrumented with the pressure, water level as well as the gas composition and temperature sensors in several positions. Similarly, the DWV instrumentation provides detailed information about the conditions inside the vessel. Since the FPV houses the EC and CCC passive systems and monitoring the conditions inside the vessel is essential for drawing the correct conclusions regarding operation of the two systems, the FPV is equipped with the largest number of measurement sensors (figure 11).

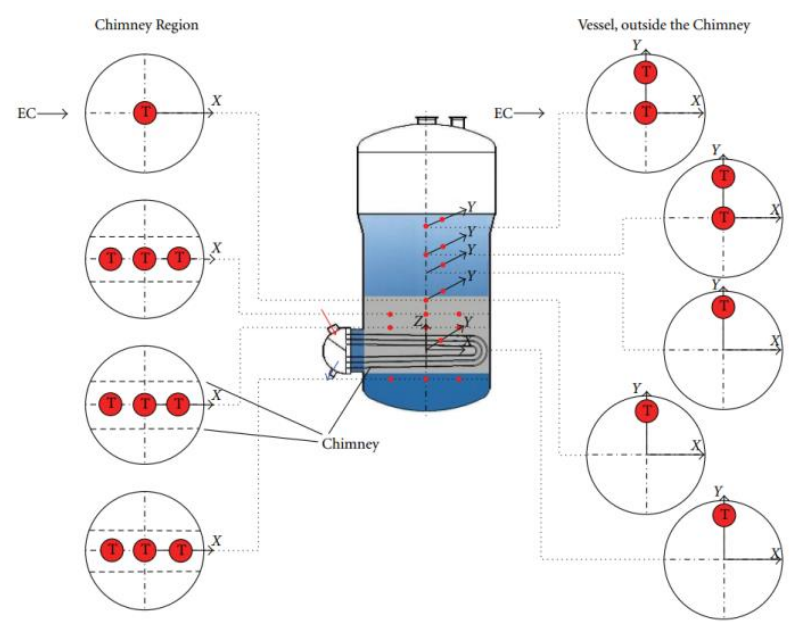

Fig. 11. Water volume instrumentation of the FPV [3]

The temperature of the water pool is measured at nine elevations. At each level up to three sensors are installed to provide an overview of the cross-sectional temperature distribution. Apart from temperature, the water level and pressure inside the vessel are measured. However, due to the overflow pipe connecting the FPV with the PSP, the water inventory cannot exceed a certain level. For investigation of the conditions around the $\mathrm{CCC}$, the space above the water in the FPV is also intensively instrumented. This region is particularly important in the light of the gas composition influence on the heat transfer capacity of the CCC.

The interface between the containment vessels and the RPV is the Emergency Condenser. The tube bundle of the EC is submerged in the water of the FPV and its inlet and outlet pipes are connected to the standpipe representing the downcomer of the KERENA RPV. The standpipe and the RPV are extensively instrumented with temperature, absolute pressure and mass flow sensors. Furthermore, a number of differential pressure measurements is installed both, in the vessels and in the EC piping for provision of accurate data regarding pressure drops and water levels. Two outer EC tubes are also densely instrumented with temperature sensors and thermo-needle probes. Most of the thermocouples are located at the axis of the pipes. Additionally, at several positions four supplementary sensors are located at the inner and outer sides of the tubes. Such a combination of the local void fraction with the temperature in five points per location enables a detailed description of the system.

Similarly to the EC, the CCC is densely instrumented for obtainment of the detailed description of the component performance. Thus, apart from the absolute pressure, the differential pressure measurements are installed at the inlet and outlet lines as well as at the heat exchange section. Thermocouples are located at several 
positions over different cross sections. The two-phase flow can be directly identified via the gamma densitometer installed in the return line.

\section{Test results}

The test program named Integral Experimental and Analytical Safety Cases for Design-Basis Accidents in Nuclear Power Plants Featuring Passive Safety Systems (EASY) was initiated in March 2015. In the frames of the project four tests were performed simulating LOCA and non-LOCA scenarios. In particular, the main steam line break, feedwater line break, station blackout and RPV bottom leak tests were performed. In this paper the main steam line break will be discussed and the performance of each passive system will be analyzed in details.

\subsection{Test boundary conditions}

During the conditioning phase of the test the INKA facility was pressurized up to 75 bars and heated up to corresponding saturation temperature. These conditions are consistent with the normal BWR operation.

In order to assure the faithful simulation of conditions inside the facility, the test comprised several activities. The break initiation was performed $350 \mathrm{~s}$. after the Start of the Test (SOT). Simultaneously, cooling of the Shielding/Storage Pool Vessel (SSPV) was initiated. This activity was necessary because the SSPV is relatively small in comparison to other components of the facility. Therefore, in order to provide SSPV heat capacity appropriately scaled with reference to the original plant, the vessel was additionally cooled.

Simultaneously, the decay heat simulation was initiated by enabling the steam flow from Benson boiler to the RPV/GAP vessel. At the same time corresponding amount of liquid was discharged from the vessel so that the latent heat of water was supplied to the system with possibly low change of the water inventory.

\subsection{Primary side}

The primary side of the INKA test facility consists of the RPV-simulator, the Standpipe, the PPPT and the EC including inlet and outlet lines.

Initiation of the break caused a rapid decrease of the pressure at the primary side. Correspondingly, the pressure at the secondary side, i.e. in the containment vessels increased. The pressures were equalized approximately $2330 \mathrm{~s}$ after the SOT. Figure 12 shows the pressures both at the primary and secondary side.

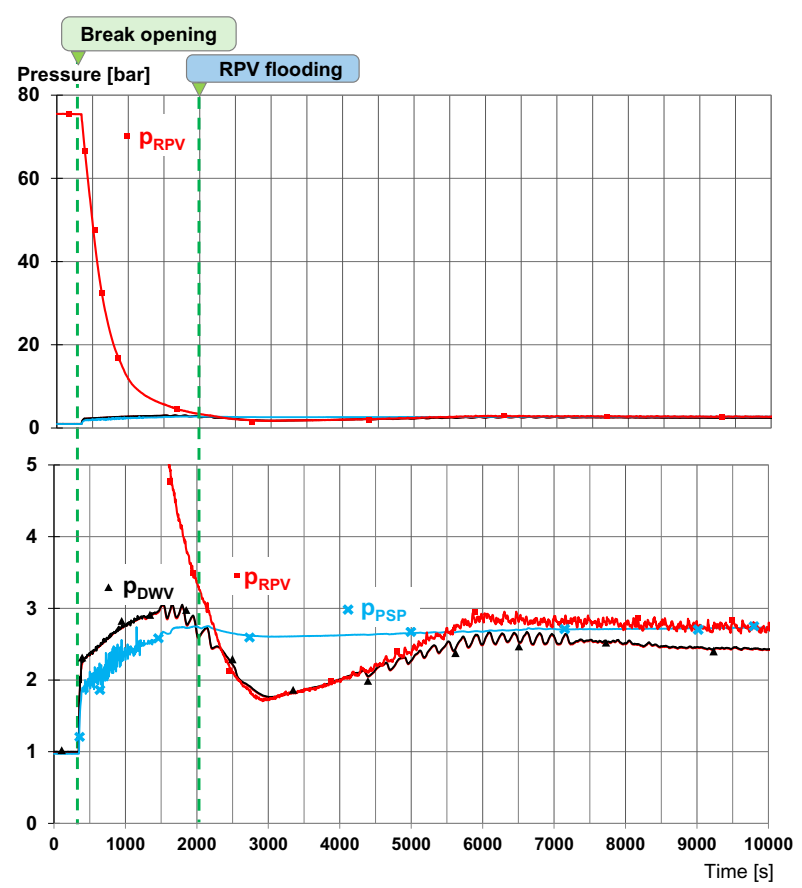

Fig. 12. Pressures in the RPV, DWV and PSP

Since during the normal operation the RPV is filled with medium under saturation conditions, rapid depressurization of the vessel leads to intensive flash boiling at the primary side. This in turn results in a loss of liquid water inventory and the fill level drop. Figure 13 depicts the water levels in the RPV and standpipe together with the dashed line illustrating the uppermost, middle and lowermost levels of the EC. The uppermost level of the EC heat exchange section is $14.3 \mathrm{~m}$. This means that once the water level decreased to this value, the EC was triggered.

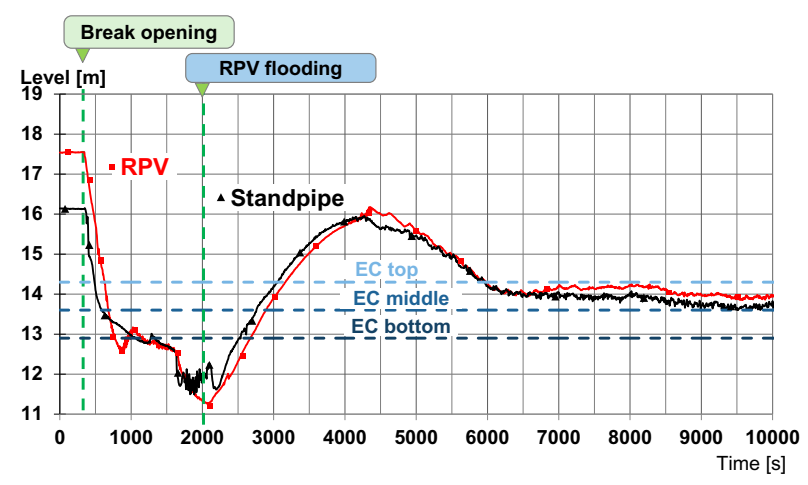

Fig. 13. Water levels in the RPV and Standpipe

In the wake of the depressurization of the system, the temperature decreased too. Shortly after the SOT, the steam and liquid temperature declining was following the saturation temperature curve. As the depressurization proceeded, the steam temperature deviated from the saturation curve leading to a significant difference after several hundred seconds. This disparity was caused by the heat transfer between hot vessel structure and steam (figure 14). 


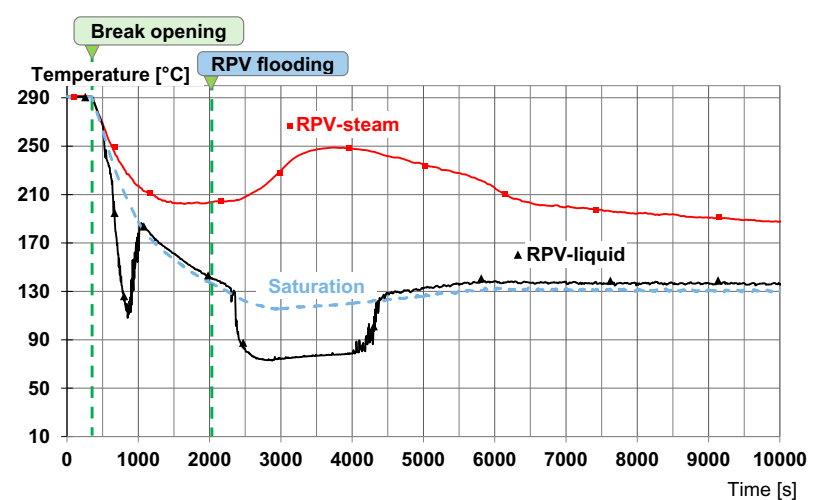

Fig. 14. RPV temperatures

In contrast, the liquid temperature followed the saturation curve most of the time. Two quick and significant drops of the liquid temperature correspond to injections of cold water. The first injection came from the liquid water occupying the EC piping during normal operation. Once this cold inventory reached the RPV, it caused a meaningful temperature decrease. Since the decay heat was being still provided to the RPV, the temperature drop was equalized within approximately $500 \mathrm{~s}$. The second decrease of the liquid water temperature was caused by triggering of the core flooding at $2062 \mathrm{~s}$ after the SOT. As the flooding flow stagnated between 4000 and $4500 \mathrm{~s}$, the liquid inventory was heated up again to saturation temperature due to the decay heat.

Figure 13 indicates that the EC was activated at $\mathrm{t}=426 \mathrm{~s}$, so about $75 \mathrm{~s}$ after the break initiation. Due to the RPV inventory loss, the water from the EC system was drained into the RPV. Consequently, the steam from the RPV entered the EC system. Figure 15 shows the measured mass flow together with the calculated power of the EC.

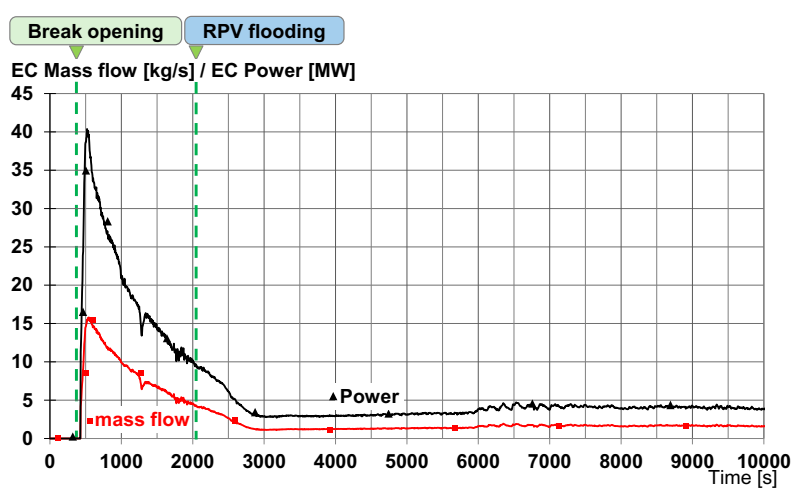

Fig. 15. EC mass flow and power

The EC power was calculated on the basis of the mass flow and the inlet and outlet enthalpies. The maximum power of $41 \mathrm{MW}$ was reached shortly after the break initiation. As the test was proceeding the power was steadily decreasing to $4 \mathrm{MW}$ and this value was kept in the long term.

Both, figure 13 and 14 are consistent with respect to the RPV flooding. Triggering of the flooding at $2062 \mathrm{~s}$ resulted in immediate increase of the fill level and slightly delayed decrease of temperature. In fact the inventory level was increased above the uppermost level of the EC piping. This means that the EC piping was completely covered with liquid water, condensation did not occur anymore and the flow through the EC should be decreased to zero. However, the condensation in the inlet pipe (between the Standpipe and the EC heat exchange section) was maintained and a low mass flow could still be observed it the EC system.

The temperature distribution in the EC piping strictly corresponded to the conditions in the other parts of the primary side. Figure 16 illustrates the temperatures at the inlet and outlet of the EC system.

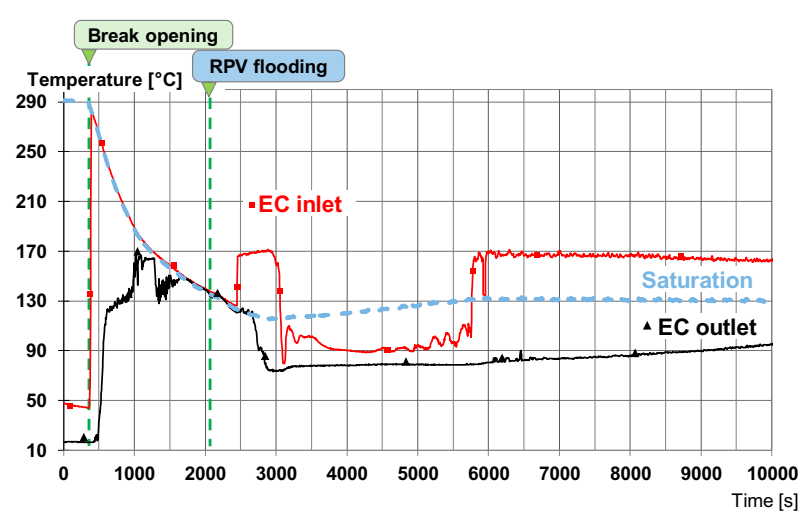

Fig. 16. EC temperatures

The start of the EC operation was indicated by the sharp increase of temperature in the top pipe row of the EC piping system from $44{ }^{\circ} \mathrm{C}$ to the saturation temperature. At this early stage the amount of condensate was not enough to cause a significant mass flow from the EC to the RPV. As the RPV inventory level was decreasing more steam entered the EC piping leading to enlarging of the EC piping uncover and the heat transfer area. This in turn led to more intensive condensation and larger mass flow through the EC.

Consistently with the RPV steam region, the temperature in the EC inlet line was also higher than the saturation temperature. Figure 16 shows also that after initiation of the RPV flooding the temperatures drop to the subcooled region. This comes from the fact that due to the equalization of the liquid levels the steam from the RPV does not enter the EC heat exchange section anymore. Therefore, EC acts in this case as a water cooler which leads to establishing of the inlet/outlet temperature difference of approximately $15 \mathrm{~K}$. After the end of the flooding and corresponding later decrease of the RPV liquid level (due to the evaporation cause by the decay heat), steam entered again the EC piping.

\subsection{Secondary side}

The secondary side of the INKA facility simulates the reactor containment and it consists of the FPV, DWV, PSPV, the vent pipe, the hydrogen overflow pipe and the siphon.

The bottom part of the figure 12 illustrates pressures at the secondary side. After the break opening an instant growth of secondary side pressure was observed. The pressure in the DWV and in the FPV increased to 3.1 bar whereas in the PSPV up to 2.7 bar. The difference of 
around 0.4 bar corresponds to the submerging of the vent pipe in the PSP water inventory.

Once the pressure at the secondary side reached its peak of 3.1 bar at $t=1600 \mathrm{~s}$, it decreased to 1.7 bar within approximately $1400 \mathrm{~s}$. Figure 12 shows that this decrease was accompanied by significant oscillations at the initial state. Both, the decrease and the oscillations are caused by the operation of the CCC. Additionally, the RPV flooding deepened the pressure decrease since it limited boiling in the RPV. Approximately $2160 \mathrm{~s}$ after the SOT, the pressures at the primary and secondary side equalized. For a certain amount of time the pressure in the RPV was even lower than in the containment vessels. This inversed difference was partly caused by injection of the cold water from the flooding system that hindered flash boiling and lowered the swell level which contributed to the steeper pressure decrease. The pressure decrease in the RPV was also supported by the condensation in the EC.

In the long term, the primary/secondary side pressure difference of around 0.3 bar was established until the end of the test. A rough estimation showed that the pressure loss of the pipe break was in this range.

Together with the pressure the temperature in the containment vessels rose sharply after the break opening. Figure 17 shows the temperatures in the INKA vessels.

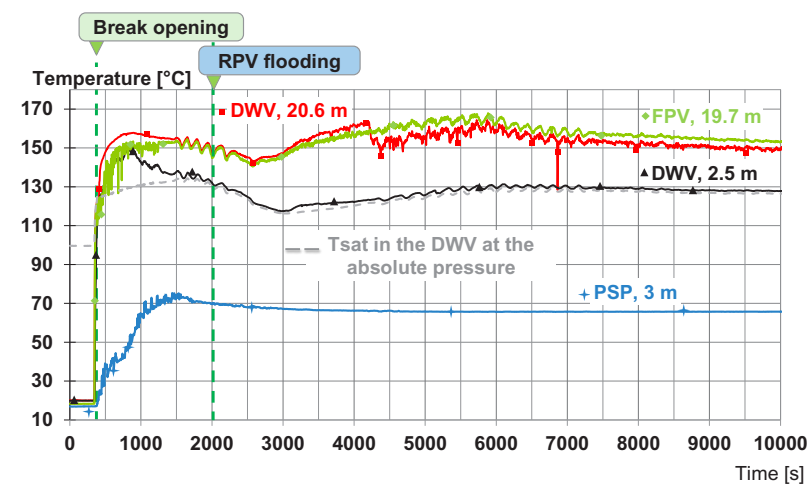

Fig. 17. DWV , FPV and PSP temperatures

Figure 17 shows that the PSP water temperature (PSP, $3 \mathrm{~m}$ ) grew steadily to approximately $65{ }^{\circ} \mathrm{C}$ and this temperature was kept until the end of the test. Due to the non-condensable gas present in the DWV and FPV more complex phenomena were observed in the gas regions of these vessels.

At the very beginning of the transients similar peaks of temperature were measured in both vessels at all elevations. As the experiment proceeded, the temperature at the bottom of the DWV $(2.5 \mathrm{~m})$ decreased to the saturation point calculated for the total pressure and it remained at this level. At the upper level of the DWV the temperature was higher and its general trend followed the conditions in the steam region in the RPV, i.e. similarly to the RPV steam (Figure 14), the temperature in the DWV started to increase around 2500 s. On the other hand, mixing with cold inert gas and pressure oscillations caused by CCC operation also had a significant influence on the final shape of the DWR temperature curve.
The temperature in the FPV shows a similar characteristics. It should be emphasized that although the medium inside the vessel was constantly and intensively mixing, the curves have rather local character. It especially refers to the upper region of the FPV, where the steam condenses at the CCC tubes. Steam condensation decreases the partial pressure of vapor and hence the total pressure too. Furthermore, the steam condensation rate depends not only on the conditions in the FPV but also in the CCC piping. Upper plot of the figure 18 shows the mass flow in the CCC system.

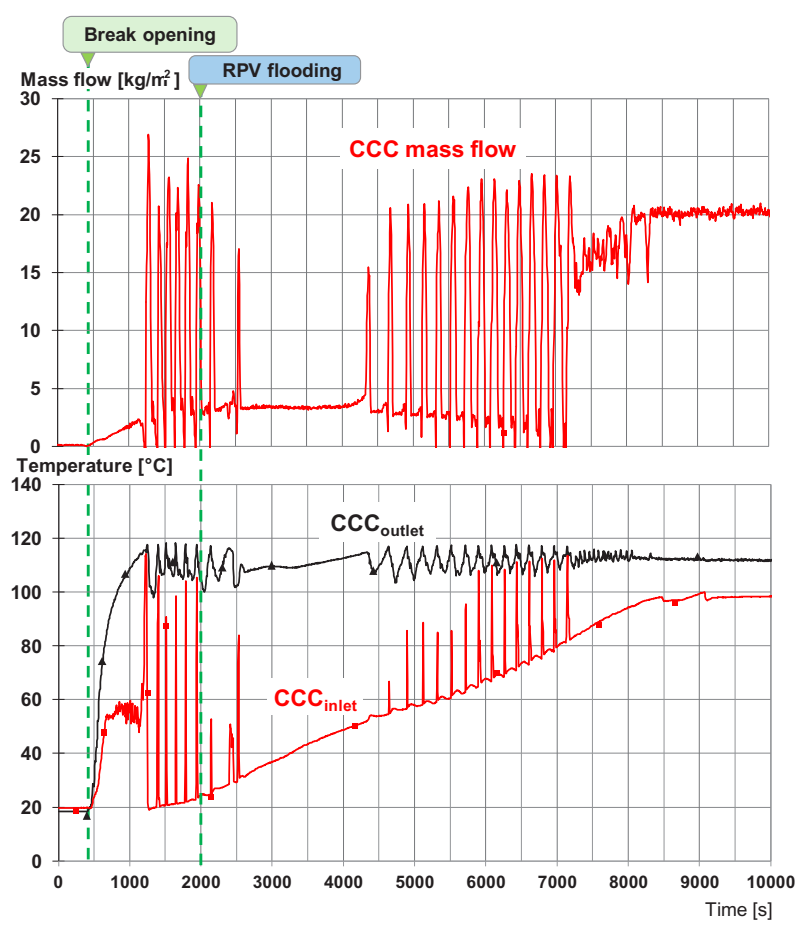

Fig. 18. CCC mass flow (top) and temperatures (bottom)

Figure 18 shows shortly after the break opening (until approximately $1200 \mathrm{~s}$ ) the mass flow was steadily increasing. During this time the cold water in the CCC was being heated up and it was naturally circulating through the CCC piping. As more and more heat was being transferred from the FPV, the water temperature increased. As the temperature reached the saturation temperature, bubbles started to form and migrate along the CCC piping. The CCC piping is only slightly inclined with respect to horizontal orientation and at the end of the heat exchange section there is a collector which enables the vertical flow. Once the bubbles migrated to this region strong buoyancy force contributed to increase of flow and displacement of the bubble to the SSPV vessel, where it condensed again. At the same time cold water was sucked by the inlet tube decreasing the temperature in the CCC piping. Due to the insignificant inclination of the CCC tubes, the reverse direction of the mechanism is also possible. Since the mass flow sensors measure the flow only in one direction, the reverse flow could not be measured, but it can be unquestionably confirmed by the inlet temperature measurement (figure 18 bottom).

The inlet and outlet tubes are connected to the collectors which then distributes or collect water to/from 
the CCC piping. Therefore, the temperatures in the figure 18 show only the overall and predominant effects (normal or reverse flow). Flow conditions in single tubes can be different and they can depend on conditions (e.g. gas composition) at the secondary side. This shows the complexity of the whole CCC system.

\section{INKA test results interpretation and benefits}

The postulated LOCA due to the main steam line break caused rapid depressurization of the RPV and a pressure increase in the vessels simulating the containment of a BWR plant. The passive safety systems investigated during the experiment reacted automatically and immediately. The first system employed for mitigation of the LOCA results was the Emergency Condenser. The most important outcomes from the EC performance are:

- The system reacts immediately to the RPV inventory change and it can be utilized as a safety injection in the whole range of pressure

- It significantly contributes to the depressurization of the primary side. This in turn lowers the pressure difference between the primary and the secondary side in case of the LOCA and it indirectly limits the pressure growth at the secondary side

- The power of the EC is large enough to remove the decay heat with substantial safety margins

The increase of pressure in the containment vessels was also limited by the PSPV. After reaching the pressure difference between the DWV and PSPV of approximately 0.4 bar, the medium from the DWV was discharged to the PSP limiting further increase of the Drywell pressure.

Simultaneously, the passive CCC system was activated contributing to the further decrease of pressure in the containment. The superposition of these systems allowed maintaining the pressure in the containment far below the design limit. Apart from pressure limitation, the CCC was proven as a reliable system for passive, long-term heat removal.

The overall test results prove that the investigated passive systems fulfill their design goals. Each of the systems is characterized with safety margins significantly exceeding the sufficient level. Thus, the whole system can be considered as redundant.

Apart from investigation the passive systems, the experimental data obtained during the test campaign were utilized for system codes validation $[5,6]$.

\section{Conclusions and outlook}

INKA is a large-scale test facility replicating an innovative BWR KERENA design of Framatome. It comprises the all passive systems of the original plant and it may be utilized for demonstration of the reference plant performance as well as for investigation of passive systems which can be applied to other LWRs.

For this reason EASY project was conducted at the INKA facility. In the frames of the project simulation of the main steam line break was performed in order to investigate the integral response of the system.
The results of the experiment confirmed the functionality of each component as well as demonstrated their redundancy. They also enabled a detailed analysis of the interactions between the systems and they contributed to better understanding of the functioning of each component integrated into the large system. Furthermore, the experimental database was utilized for thermal-hydraulic codes validation.

In total four tests were performed in the framework of the EASY_1 project. Further investigations are planned to be conducted as the EASY_2 continuation project.

\section{References}

1. Stosic Z., Brettschuh W., Stoll U, Boiling water reactor with innovative safety concept: The Generation III+ SWR-1000, Nuclear Engineering and Design Vol. 238, pp. 1863 - 1901, (2008).

2. Bryk R., Schmidt H., Mull T., Wagner T., Ganzmann I., Herbst O., Modeling of KERENA emergency condenser, Archives of Thermodynamics Vol. 38, no. 4, pp. 29 - 51, (2017).

3. Leyer S., Wich M., The Integral Test Facility Karlstein, Science and Technology of Nuclear Installations, Vol. 2012, (2012).

4. Leyer S., Wich M., Schafer H., SWR 1000 integral and full scale tests of the passive safety systems, Proceedings of the International Conference on Advances in Nuclear Power Plants (ICAPP '08), pp. 87 - 92, (2008).

5. Drescher R., Wagner T., Prasser H. M, Leyer S., Passive Integral LOCA Testing at Karlstein Test Facility INKA, Proceedings of the ICAPP 2014, (2014).

6. Bryk R., Schmidt H., Mull T., Modeling of emergency condenser system response to loss of coolant accident in a BWR III+ generation, Eksploatacja i Niezawodność - Maintenance and Reliability, Vol. 21, no. 3, pp. 468 - 475 (2019). 